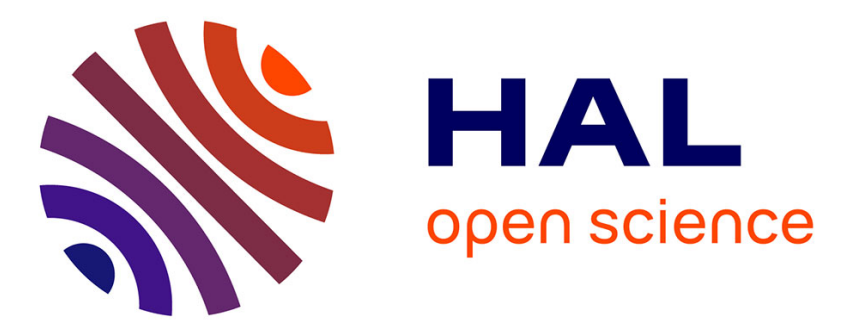

\title{
Influence du pH du milieu au cours de la fécondation sur la survie embryonnaire de la carpe commune (Cyprinus carpio)
}

Pierre Roubaud, Christian Gillet, Roland Billard

\section{- To cite this version:}

Pierre Roubaud, Christian Gillet, Roland Billard. Influence du pH du milieu au cours de la fécondation sur la survie embryonnaire de la carpe commune (Cyprinus carpio). Canadian Journal of Zoology, 1984, 62 (5), pp.851-861. 10.1139/z84-125 . hal-02728586

\section{HAL Id: hal-02728586 \\ https://hal.inrae.fr/hal-02728586}

Submitted on 2 Jun 2020

HAL is a multi-disciplinary open access archive for the deposit and dissemination of scientific research documents, whether they are published or not. The documents may come from teaching and research institutions in France or abroad, or from public or private research centers.
L'archive ouverte pluridisciplinaire HAL, est destinée au dépôt et à la diffusion de documents scientifiques de niveau recherche, publiés ou non, émanant des établissements d'enseignement et de recherche français ou étrangers, des laboratoires publics ou privés.

\section{다(1)(2)}

Distributed under a Creative Commons Attribution - ShareAlikel 4.0 International 


\title{
Influence du pH du milieu au cours de la fécondation sur la survie embryonnaire de la carpe commune (Cyprinus carpio)
}

\author{
P. ROUBAUD \\ Station centrale de physiologie animale, Groupe poissons, Institut national de la recherche agronomique, \\ F 78350 Jouy en Josas, et Université Paris VII, Equipe de biologie du développement, Paris, France \\ ET \\ Ch. Gillet et R. Billard \\ Station centrale de physiologie animale, Groupe poissons, Institut national de la recherche agronomique, \\ F 78350 Jouy en Josas, France. \\ Reçu le 5 juillet 1983
}

\begin{abstract}
Roubaud, P., CH. Glllet et R. Billard. 1984. Influence du pH du milieu au cours de la fécondation sur la survie embryonnaire de la carpe commune (Cyprinus carpio). Can. J. Zool. 62: 851-861.

Les effets du pH dans le milieu pendant la fécondation de l'oeuf de carpe sont étudiés en mesurant le pourcentage de survie embryonnaire avant éclosion. Ces effets, qui peuvent être différés au delà de l'épibolie, sont les mêmes pour des durées de traitement de 15 et $30 \mathrm{~min}$ à partir de l'insémination et ne sont modifiés ni par unc adjonction au milieu de solution salinc physiologique, ni par une exposition des ovules pendant $10 \mathrm{~s}$ avant l'insémination. Parmi les six espèces d'agent tampon utilisées, les phosphates manifestent une toxicité particulière qui traduit peut-être le rôle joué par le calcium lors de la fécondation. Une fécondation normale exige une osmolarité du milieu inférieure ou égale à $200 \mathrm{mosmol} / \mathrm{kg}$. Au delà des limites 7,2 et 9,6 de l'intervalle de $\mathrm{pH}$ favorable, la tolérance de l'ocuf décroit moins vite dans un milieu faiblement tamponné que dans un milieu fortement tamponné. Dans un tel milieu, elle s'annule aux pH 6,2 ou 9,9.
\end{abstract}

Roubaud, P., Ch. Gillet, and R. Billard. 1984. Influence du pH du milieu au cours de la fécondation sur la survie embryonnaire de la carpe commune (Cyprinus carpio). Can. J. Zool . 62: $851-861$

The effects of environmental $\mathrm{pH}$ during fertilization in carp eggs were studied by measuring the percentage of prehatching embryonic survival. These effects, which may be delayed beyond epiboly, were the same when the treatments lasted 15 and 30 min after insemination, and they were not modificd either by the addition of a physiological salt solution to the medium or by exposure of the eggs for $10 \mathrm{~s}$ before insemination. Among the six types of buffer used, phosphate buffers were particularly toxic which may indicate the role played by calcium during fertilization. Normal fertilization requires that the osmolarity of the medium be lower than or equal to $200 \mathrm{mosmol} / \mathrm{kg}$. Beyond the limits of favorable $\mathrm{pH}$ values (7.2 and 9.6 ), egg tolerance decreased less rapidly in a weakly buffered medium than in a strongly buffered one. In such a medium, there was no resistance at $\mathrm{pH} 6.2$ or 9.9 .

\section{Introduction}

La détermination des conditions optimales et des conditions limites de survie et de développement pour les stades gamétiques et embryonnaires précoces des poissons d'eau douce présente un intérêt à la fois fondamental et appliqué.

En pisciculture, le facteur déterminant de la réussite ou de l'échec se trouve, non dans la qualité moyenne du milieu offert aux animaux, mais dans l'amplitude et le moment des écarts extrèmes, souvent accidentels, qui leur sont imposés. Cette remarque vaut aussi pour les poissons vivant dans le milieu naturel et soumis aux ćcarts naturels des paramètres physicochimiques et biologiques de l'environnement et à des pollutions artificielles de plus en plus diverses. De ce point de vue, pensons nous, et plus encore qu'en pisciculture, les phases gamétiques et le début du développement embryonnaire précoce sont les plus importants à connaître parce qu'ils apparaissent souvent comme plus sensibles que les autres phases du cycle vital (McKim 1977; Jaoul et Roubaud 1982; Gillet et Roubaud 1983; Billard et Roubaud 1984). C'est ainsi qu'ont été mis en évidence pour plusieurs espèces d'importants changements de la tolérance aux bas pH au cours de l'ontogénèse. La fragilité particulière des embryons et alevins de Salmo salar permet par exemple d'expliquer leur absence dans les rivières où les adultes sont encore présents (McKim 1977). Dans cette même espèce, la sensibilité aux bas $\mathrm{pH}(s)$ peut être classée suivant les stades du développement embryonnaire (Daye et Garside 1977, 1979): $s$ (jeune alevin) $>s$ (embryon en segmentation) $>s$ (embryon oeillé). De même, pour l'oeuf de Salmo trutta: $s$ (embryon en segmentation) $>s$ (embryon oeillé) et $s$ (jeune alevin) $>s$ (embryon oeillé) (Brown et Lynan 1981). Pour l'espèce Jordanella floridae: $s$ (ovogénèse) $>s$ (alevin) $>s$ (embryon pré-éclos) (Craig et Baksi 1977). Cependant l'interprétation de Craig et Baksi (1977), estimant que la grande résistance aux $\mathrm{pH}$ acides s'étend sur la totalité du développement embryonnaire précoce, est critiquée par Lee et Gerking (1980) qui montrent, sur Cyprinodon n. nevadensis, que l'on doit distinguer dans l'embryogénèse précoce une période de sensibilité particulière au moment de l'activation. De même, l'embryon de Perca fluviatilis est particulièrement sensible aux eaux acides avant l'âge de $24 \mathrm{~h}$ (Johanson et Milbrink 1976). Les recherches effectuées en vue de réaliser, pour Salmo irideus, un dilueur d'insémination artificielle (DIA) ont montré que l'optimum de $\mathrm{pH}$ se situe au voisinage de $\mathrm{pH}$ 9. En dessous du $\mathrm{pH} 7$, les pourcentages de fécondation sont plus faibles (Petit et al. 1973). De même, sur le brochet (Esox lucius), le DIA le plus favorable a un pH 9 (Marcel 1981). Ces résultats tendent à orienter la recherche des périodes critiques de sensibilité au facteur de $\mathrm{pH}$ vers la fécondation et les tout cébuts du développement embryonnaire, stades pour lesquels les données manquent quant à l'acidorésistance.

Nous avons entrepris l'étude de l'acidotolérance et de alcalinotolérance de l'oeuf de la carpe commune (Cyprinus carpio L.), espèce d'intérêt économique de large distribution géographique et qui présente des avantages importants pour les études d'embryologie expérimentale (Jaoul et Roubaud 1982): nombre d'oeufs pondus, facilité de l'incubation, transparence 
de la coque et possibilité d'obtenir simplement des oeufs pendant toute l'année.

\section{Matériels et méthodes: modèle biologique}

L'expérimentation a consisté à réaliser la fécondation dans des milieux simples à base de $\mathrm{NaCl}$ ou enrichis en divers ions minéraux et tamponnés dans une large gamme de $\mathrm{pH}(4,75-10,6)$. Après fécondation, les oeufs sont laissés pendant $30 \mathrm{~min}$ ( $15 \mathrm{~min}$ dans l'expérience 2B) dans ces mêmes milieux, c'est-à-dire approximativement jusqu'au moment de la fusion des pronucleus mâle et femelle. Les effets du traitement sont appréciés par la mesure des pourcentages de survie à divers stades du développement embryonnaire: stade du bourgeon caudal, stade oeillé, stade de pré-éclosion.

\section{Production des gametes}

Les reproducteurs, carpes de variété miroir, sont élevés sous photopériode et thermopériode naturelles, dans une eau recyclée dont les propriétés thermiques et physicochimiques sont contrôlées. Les femelles âgées de 6 à 7 ans effectuent leur $3^{\mathrm{e}}$ ou $4^{\mathrm{e}}$ cycle reproducteur. L'hypophysation est pratiquée dvec $3 \mathrm{mg} / \mathrm{kg}$ de poids vif d'extraits hypophysaires bruts de carpe (Stoller Fisheries, activité équivalente à $63 \mu \mathrm{g}$ d'hormone gonadotrope selon le dosage réalisé par la technique de maturation ovocytaire in vitro de l'ovocyte de truite (Jalabert et al. 1974)), en novembre et décembre 1980 (expériences 2, 3A et 3B), en janvier, juillet et août 1981 (expériences 1A, 4 et 5), en mars 1982 (expériences $1 \mathrm{~B}$ et 6 ) et en décembre 1982 pour l'expérience 7. Lorsque la température d'élevage est inférieure à $15^{\circ} \mathrm{C}$, les femelles sont placécs 15 jours avant l'hypophysation à la température de $20^{\circ} \mathrm{C}$. Les ovules de chaque femelle sont récoltés séparemment par pression abdominale et rapidement fécondés par un mélange de sperme provenant de trois mâles placés au préalable pendant quelques jours en eau à $20^{\circ} \mathrm{C}$ et recevant une injection de $1 \mathrm{mg} / \mathrm{kg}$ de poids corporel du même extrait hypophysaire que celui utilisé pour les femelles.

\section{Insemination}

Dans toutes les expériences (sauf l'expérience 4, série A1), les ovules, prélevés par lots d'environ 200 à l'aide d'une spatule sèche, sont dispersés simultanément avec $100 \mu \mathrm{L}$ de sperme déposé à la microseringue (Eppendorf), dans une boite de Petri contenant $20 \mathrm{~mL}$ de DIA équilibré à la température de $20^{\circ} \mathrm{C}$. Dans l'expérience 4 , série $\mathrm{Al}$, les ovules sont dispersés dans lc DIA $10 \mathrm{~s}$ exactement avant l'introduction de la goutte de sperme qui est alors dispersée par agitation pendant $15 \mathrm{~s}$

Dans les secondes qui suivent leur introduction dans le DIA (sauf aux pH les plus alcalins, ou lorsque l'osmolarité du DIA dépasse 300 mosmol), les oeufs se collent sur le fond de la boite de Petri; on évite par agitation, qu'ils ne s'agglutinent, ce qui limiterait leur oxygénation au cours de l'incubation. A partir de la $15^{\mathrm{e}}$ min dans l'expérience $2 \mathrm{~A}$ et à partir de la $30^{\mathrm{e}}$ min dans toutes les autres expériences, les oeufs sont rincés trois fois en $0.25 \mathrm{~h}$ avec de l'eau du robinet de température à $20^{\circ} \mathrm{C}$

\section{Incubation}

Les oeufs sont incubés à la température de $20^{\circ} \mathrm{C}$. Dans les expériences 2 et 3 , les oeufs sont placés sous une couche d'eau du robinet de $5 \mathrm{~mm}$ d'épaisseur renouvelée toutes les $12 \mathrm{~h}$. Dans les expériences $1 \mathrm{~A}, 1 \mathrm{~B}, 4,5,6$ et 7 , l'incubation a lieu simplement à l'air humide.

\section{Mesure des mortalités}

L'effet des traitements est évalué par le pourcentage des oeufs vivants: le critère de reconnaissancc d'un ocuf mort est l'opacité de son vitellus. Sauf dans l'expérience 3B, où les comptages sont effectués aux âges de 30 et $48 \mathrm{~h}$, les pourcentages de survie sont mesurés dans l'intervalle $68-72 \mathrm{~h}$, c'est-à-dire, pour un développement normal, peu avant le début de l'éclosion.

\section{Protocoles expérimentaux, composition des milieux d'insémination} artificielle

Pour la réalisation des dilueurs d'insémination, on a considéré le pH, l'osmolarité, le pouvoir tampon, la toxicité éventuelle des espèces chimiques utilisées comme agent tampon de $\mathrm{pH}$, ct la concentration en ions minéraux fondamentaux $\left(\mathrm{Cl}^{-}, \mathrm{Na}^{+}, \mathrm{K}^{+}, \mathrm{Ca}^{2+}\right)$. Ces différents paramètres ne sont pas indépendants, en particulier on ne peut obtenir un pouvoir tampon élevé sans augmenter l'osmolarité du dilueur. Les DIA ont été préparés à partir de solutions mères molaires de trois bases et trois acides. Les trois bases sont 4,3-aminopropylmorpholine (APM) (solution $M$ ), l'ethylène diamine (EDA) (solution $E$ ) et le Tris(hydroxyméthyl)aminométhane (Tris) (solution T); les trois acides sont l'acide acétiquc (solution $A$ ), l'acide phosphorique (solution $P$ ) et la glycine (solution $\mathrm{G}$ ).

Ces espèces chimiques admettent comme $\mathrm{pK}$ d'ionisation dans l'eau les valeurs respectives suivantes (Von Rauen 1964): APM, 10,2 ct 6,2; EDA, 9,9 et 6,8; Tris, 8,2; l'acide acétique, 4,75; l'acide phosphorique, 7,$2 ;$ la glycine, 9,6 . Les solutions mères, traitées par l'acide chlorhydrique normal ou la soude normale pour ajuster leur $\mathrm{pH}$ à la valeur voulue, ont été diluées soit par l'eau bidistillée (expériences $1 \mathrm{~A}$ et 3 ), soit par une eau du robinet (expériences 1B, 2, 4, 5, 6 et 7)

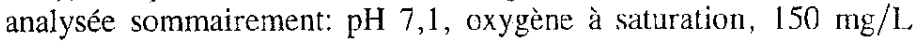
$\mathrm{CO}_{3} \mathrm{Ca}$ et traces de $\mathrm{NH}_{4}^{+}, \mathrm{NO}_{3}^{-}$et phosphates. Dans les expériences 1 ct 3 , un complément d'ions minéraux a été apporté par adjonction d'une solution saline, physiologiquement équilibrée (solution $\mathrm{H}$ ), développant une osmolarité de $1 \mathrm{osmol} / \mathrm{kg}$ et contenant, pour $1 \mathrm{~L}$ de solution: $\mathrm{NaCl}, 28,3 \mathrm{~g} ; \mathrm{KCl}, 0,5 \mathrm{~g} ; \mathrm{CaCl}, 1,1 \mathrm{~g}$. Les $\mathrm{pH}$ ont été contrôlés avec un appareil Metrohm Merisau E 532 et les osmolarités mesurées par abaissement du point de congélation avec un appareil Vogel 6306.

Expérience 1. Influence de l'osmolarité du DIA sur la survie embryonnaire avant éclosion

Dans une première série de tests (expérience 1A), des solutions tamponées développant des osmolarités de 5, 50, 149, 200, 247, 345 et $493 \mathrm{mosmol} / \mathrm{kg}$ au moment de l'emploi ont été obtenues en diluant à $1 \mathrm{~L}$ par de l'eau bidistillée respectivement $5,50,150,200,250$, 350 et $500 \mathrm{~mL}$ de solution $\mathrm{H}$.

Dans une deuxième série de tests (expérience 1B), des solutions tamponnées ont été préparées en diluant par l'eau du robinet la solution mère $\mathrm{T}$ portée au pH 8,2 par une solution molaire de $\mathrm{HCl}$, jusqu'à l'obtention d'une osmolarité finale de $100 \mathrm{mosmol} / \mathrm{kg}$. A cette solution, la quantité nécessaire de glycérol ou de $\mathrm{NaCl}$ ou de chlorure de choline á été ajoutée pour obtenir des osmolarités finales de 150,200, $250,300,350$ et $400 \mathrm{mosmol} / \mathrm{kg}$.

Expérience 2 (tableau 1). Détermination de l'intervalle de $\mathrm{pH}$ favorable, (IpH-F), avec les ions phosphate, Tris et glycine, en présence de sels minéraux. Effels de la dilution du DIA. Comparaison de deux durées de traitement

Sept valeurs de pH ont été essayées: 6,2 et 7,2 avec l'ion phosphate, $7,2,8,2$ et 9,2 avec l'ion Tris et 8,6 et 10,6 avec l'ion glycine. Pour chacun de ces ions et chacune de ces valeurs de $\mathrm{pH}$, des solutions dilućes au $1: 3$ (solutions $P_{b}, T_{b}$ et $G_{b}$ ) et des solutions diluées au $1: 7,5$ (solutions $P_{c}, T_{c}$ et $G_{c}$ ) ont été préparées à partir de solutions concentrées d'osmolarité $150 \mathrm{mosmol} / \mathrm{kg}$ (solutions $\mathrm{P}_{\mathrm{a}}$, $\mathrm{T}_{\mathrm{a}}$ et $\mathrm{G}_{\mathrm{a}}$ ). Ces solutions concentrées conticnnent respectivement, pour $1 \mathrm{~L}, 37,5 \mathrm{~mL}$ de solution mère $P$ ou $50 \mathrm{~mL}$ de solution mère, $T$ ou $G$; elles ont été complétées par la quantité nécessaire de solution saline $\mathrm{H}$ pour arriver à l'osmolarité de $150 \mathrm{mosmol} / \mathrm{kg}$.

Le protocole experimental a comporté la comparaison des effets sur la survie à l'éclosion de deux durées de traitement à partir de l'insémination: 15 et 30 min.

Expérience 3 (tableau 2). Action des DIA concentrés non minéralisés. Evolution de la survie au cours de l'embryogénèse

Les ions phosphate, Tris et glycine, ont été testés pour les mêmes valeurs du $\mathrm{pH}$ que dans l'expérience 2. En outre, dans l'expérience $3 \mathrm{~B}$, l'ion acétate a permis d'essayer des $\mathrm{pH}$ plus bas: $\mathrm{pH} 5$ et 6. Les concentrations en espèce tampon étaient comparables à celles de l'expérience 2 (série A), mais, en l'absence d'apport minéral, les osmolarités étaient plus faibles ct de valeurs inégales. Les pourcentages de survie ont été mesurés à l'éclosion dans l'expérience $3 \mathrm{~A}$, et aux stades de "bourgeon caudal" (âge 30 h) et "oeillé" (âge 48 h), dans l'expérience 3B. 
Tableau 1. Composition des milicux d'insémination artificielle (expérience 2)

\begin{tabular}{|c|c|c|c|c|c|}
\hline $\begin{array}{l}\text { Nature de } \\
\text { l'espèce tampon, et } \\
\text { désignation de la } \\
\text { solution mère et de } \\
\text { la série de dilution }\end{array}$ & $\begin{array}{c}\mathrm{pH} \\
\text { au moment } \\
\text { de l'emploi }\end{array}$ & $\begin{array}{c}\mathrm{pH} \\
\text { en fin de } \\
\text { traitement }\end{array}$ & $\begin{array}{l}\text { Concentration } \\
\text { en espèce chimique } \\
\text { tampon }([C]) \\
(\text { mosmol } / \mathrm{kg})\end{array}$ & $\begin{array}{c}\text { Concentration } \\
\text { en solution } \\
\text { saline }(\mathrm{H}) \\
(\mathrm{mL} / \mathrm{L})\end{array}$ & $\begin{array}{c}\text { Osmolarité } \\
(\mathrm{mosmol} / \mathrm{kg})\end{array}$ \\
\hline $\mathrm{P}_{\mathrm{a}}$ & 6,20 & 6,30 & 37,5 & 75 & 155 \\
\hline $\mathrm{P}_{\mathrm{b}}$ & 6,20 & 6,39 & 12,5 & 25 & 54 \\
\hline $\mathrm{P}_{c}$ & 6,20 & 6,46 & 5,0 & 10 & 21 \\
\hline $\mathrm{P}_{\mathrm{a}}$ & 7,20 & 7,18 & 37,5 & 56 & 151 \\
\hline$P_{b}$ & 7,20 & 7,18 & 12,5 & 18,6 & 53 \\
\hline $\mathrm{P}_{\mathrm{c}}$ & 7,20 & 7,16 & 5,0 & 57,7 & 20 \\
\hline $\mathrm{T}_{\mathrm{a}}$ & 7,20 & 7,17 & 50,0 & 55 & 150 \\
\hline $\mathrm{T}_{\mathrm{b}}$ & 7,20 & 7,02 & 16,6 & 18,3 & 50 \\
\hline $\mathrm{T}_{\mathrm{c}}$ & 7,20 & 6,90 & 6,66 & 7,3 & 22 \\
\hline $\mathrm{T}_{\mathrm{a}}$ & 8,20 & 8,05 & 50 & 75 & 150 \\
\hline $\mathrm{T}_{\mathrm{b}}$ & 8,20 & 7,88 & 16,6 & 25 & 50 \\
\hline $\mathrm{T}_{\mathrm{c}}$ & 8,20 & 7,60 & 6,66 & 10 & 22 \\
\hline$T_{a}$ & 9,20 & 8,78 & 50 & 95 & 154 \\
\hline $\mathrm{T}_{\mathrm{b}}$ & 9,20 & 8,49 & 16,6 & 31,6 & 54 \\
\hline $\mathrm{T}_{c}$ & 9,20 & 8,15 & 6,66 & 12,6 & 21 \\
\hline $\mathrm{G}_{\mathrm{z}}$ & 8,60 & 7,69 & 50 & 95 & 155 \\
\hline $\mathrm{G}_{\mathrm{b}}$ & 8,60 & 7,18 & 16,6 & 31,6 & 52 \\
\hline $\mathrm{G}_{\mathrm{c}}$ & 8,60 & 7,17 & 6,66 & 12,6 & 21 \\
\hline $\mathrm{G}_{\mathrm{a}}$ & 10,60 & 9,1 & 50 & 55 & 151 \\
\hline $\mathrm{G}_{\mathrm{b}}$ & 10,60 & 8,82 & 16,6 & 18,3 & 53 \\
\hline$G_{c}$ & 10,60 & 8,27 & 6,66 & 7,3 & 20 \\
\hline
\end{tabular}

Tableau 2. Composition des milieux d'insémination artificielle (expérience 3)

\begin{tabular}{|c|c|c|c|}
\hline $\begin{array}{c}\text { Nature de } \\
\text { l'espèce tampon } \\
\text { et désignation } \\
\text { de la solution } \\
\text { mère }\end{array}$ & $\begin{array}{c}\mathrm{pH} \\
\text { aut } \\
\text { moment } \\
\text { de l'emploi }\end{array}$ & $\begin{array}{l}\text { Concentration } \\
\text { en espèce } \\
\text { chimique } \\
\text { tampon }([C]) \\
\text { (mosmol } / \mathrm{kg})\end{array}$ & $\begin{array}{l}\text { Osmolarité } \\
(\mathrm{mosmol} / \mathrm{kg})\end{array}$ \\
\hline $\mathrm{P}$ & 6,2 & 45 & 95 \\
\hline $\mathrm{P}$ & 7,2 & 45 & 110 \\
\hline $\mathrm{T}$ & 7,2 & 50 & 86 \\
\hline $\mathrm{T}$ & 8,2 & 50 & 67 \\
\hline $\mathrm{T}$ & 9,2 & 50 & 61 \\
\hline $\mathrm{G}$ & 8,6 & 50 & 61 \\
\hline $\mathrm{G}$ & 9,6 & 50 & 74 \\
\hline $\mathrm{G}$ & 10,6 & 50 & 94 \\
\hline $\mathrm{A}$ & 5,0 & 50 & 80 \\
\hline $\mathrm{A}$ & 6,0 & 50 & 95 \\
\hline
\end{tabular}

Expérience 4 (tableau 3). Exposition simultanée des ovules et du sperme ou exposition des ovules $10 \mathrm{~s}$ avant l'insémination; détermination de l'IpH-F à deux concentrations du DIA avec apport minéral limite

Neuf valeurs de $\mathrm{pH}$ ont été testées, utilisant les ions acétate aux pH 4,75 et 5,75, phosphate aux pH 6,2, 7,2 et 8,2, Tris aux pH 7,2, 8,2 et 9,2 et glycine aux pH $8,6,9,6$ et 10,6 .

Dans une première série d'essais (série A), les concentrations choisies en espèce tampon étaient égales (pour l'acide phosphorique) ou supérieures (pour le Tris et la glycine) à celles utilisćes dans l'expérience 2 , série $\mathrm{A}$. Cependant, un apport moins important de solution saline $\mathrm{H}$ conduisait à une osmolarité plus faible. Avec l'acide acétique, une concentration très élevée en espèce tampon ( $100 \mathrm{mM}$ ) était employée pour obtenir, au pH 5,75 relativement éloigné du pK d'ionisation, un pouvoir tampon encore important.
Dans une deuxième série d'essais (série B), les effets d'une dilution au 1:5 des DIA utilisés dans la série $A$ ont été étudiés.

Enfin, avec les DIA concentrés de la série A, les effets sur la survie à l'éclosion d'une exposition des ovules de $10 \mathrm{~s}$ préalablement à l'insémination dans le DIA (serie Al) ont été comparés à ceux produits par une introduction simultanée de gamétes mâle et femelle (série A2).

Expérience 5 (tableau 3). Détermination de l'IpH-F sur les ovules séparés de deux femelles. Essais de l'EDA comme tampon

Outre les cinq valeurs de $\mathrm{pH}$ testées avec les mêmes DIA que dans l'expérience précédente, l'utilisation de l'EDA a permis d'étudier les valeurs $5,8,6,8$ et 7,8 du côté acide et $8,9,9,9$ et 10,9 du côté alcalin. Pour ces trois premières valeurs mettant en jeu la deuxième charge d'ionisation de l'EDA, les concentrations $([C])$ étaient $50 \mathrm{mM}$, ce qui permettait une comparaison avec les concentrations utilisées pour le Tris et la glycine dans l'expérience 2 (série A). Pour les trois valeurs alcalines d'utilisation de l'EDA, mettant en jeu seulement sa première charge d'ionisation, les concentrations $([C]=75 \mathrm{mM})$ étaient celles choisies pour le Tris et la glycine dans l'expérience 5. Par un apport sal in comparable à celui utilisé dans l'expérience 2 (série A), l'osmolarité était ajustée au voisinage de $150 \mathrm{mosmol} / \mathrm{kg}$.

Le protocole expérimental a permis la comparaison de la résistance des ovules produits séparemment par deux femelles après fécondation avec le même mélange des spermes produits par trois mâles.

Expérience 6 (tableau 4). Etude de la tolérance de l'oeuf aux bornes de l'IpH-F

A côté des DIA contenant des ions glycine, Tris et EDA, des DIA à l'APM ont été testés. Six valeurs de pH ont été ainsí essayées, chacune d'entre elles correspondant au $\mathrm{pK}$ d'ionisation d'une espèce tampon, c'est-à-dire au $\mathrm{pH}$ assurant un pouvoir tampon maximum de l'agent utilisé: 9,9 et 6,8 pour l'EDA, 10,2 et 6,2 pour l'APM, 8,2 pour le Tris et 9,6 pour la glycine. De façon à obtenir un pouvoir tampon maximum sans dépasser la valeur limite de $200 \mathrm{mosmol} / \mathrm{kg}$ pour l'osmolarité, les solutions ont été calculées de façon à développer sans adjonction saline minérale, une osmolarité de $150 \mathrm{mosmol} / \mathrm{kg}$. Les concentrations en espèce tampon étaient donc $100 \mathrm{~m} M / \mathrm{kg}$ pour les DIA ne mettant en jeu qu'une seule charge d'ionisation (APM au 
Tableau 3. Composition des milieux d'insémination artificielle (expériences 4 et 5 )

\begin{tabular}{|c|c|c|c|c|c|c|}
\hline \multirow{2}{*}{$\begin{array}{l}\text { Nature de } \\
\text { l'espèce tampon, et } \\
\text { désignation de la } \\
\text { solution mère et de } \\
\text { la série de dilution }\end{array}$} & \multirow{2}{*}{$\begin{array}{c}\mathrm{pH} \\
\text { au moment } \\
\text { de l'emploi }\end{array}$} & \multicolumn{2}{|c|}{$\begin{array}{l}\mathrm{pH} \\
\text { en fin de } \\
\text { traitement }\end{array}$} & \multirow{2}{*}{$\begin{array}{c}\text { Concentration } \\
\text { en espćce chimique } \\
\text { tampon }([C]) \\
(\text { mosmol } / \mathrm{kg})\end{array}$} & \multirow{2}{*}{$\begin{array}{l}\text { Concentration } \\
\text { en solution } \\
\text { saline }(\mathrm{H}) \\
(\mathrm{mL} / \mathrm{L})\end{array}$} & \multirow[b]{2}{*}{$\begin{array}{c}\text { Osmolarité } \\
(\mathrm{mosmol} / \mathrm{kg})\end{array}$} \\
\hline & & $\begin{array}{c}\text { Expérience }^{a} \\
4\end{array}$ & $\begin{array}{c}\text { Expéricnce" } \\
5 \mathrm{~A} \\
\end{array}$ & & & \\
\hline $\mathrm{P}_{\mathrm{a}}$ & 6,2 & 6,12 & \multirow[t]{2}{*}{6,10} & 37,5 & 50 & \multirow[t]{2}{*}{136} \\
\hline$P_{b}$ & 6,2 & 6,15 & & 7,5 & 10 & \\
\hline $\mathrm{P}_{\mathrm{a}}$ & 7,2 & 7,03 & \multirow[t]{2}{*}{7,09} & 37,5 & 25 & \multirow[t]{2}{*}{116} \\
\hline$P_{b}$ & 7,2 & 7,06 & & 7,5 & 5 & \\
\hline$P_{a}$ & 8,2 & 8,06 & \multirow[t]{2}{*}{8,10} & 37,5 & 25 & \multirow[t]{2}{*}{134} \\
\hline $\mathrm{P}_{\mathrm{b}}$ & 8,2 & 8,00 & & 7,5 & 5 & \\
\hline$T_{a}$ & 7,2 & 7,00 & \multirow[t]{2}{*}{7,11} & 75 & 1 & \multirow[t]{2}{*}{143} \\
\hline $\mathrm{T}_{\mathrm{b}}$ & 7,2 & 7,06 & & 15 & 0,2 & \\
\hline $\mathrm{T}_{\mathrm{a}}$ & 8,2 & 8,01 & \multirow[t]{2}{*}{7,98} & 75 & 5 & \multirow[t]{2}{*}{118} \\
\hline $\mathrm{T}_{\mathrm{b}}$ & 8,2 & 7,76 & & 15 & 1 & \\
\hline $\mathrm{T}_{\mathrm{a}}$ & 9,2 & 9,05 & \multirow[t]{2}{*}{8,90} & 75 & 50 & \multirow[t]{2}{*}{132} \\
\hline $\mathrm{T}_{\mathrm{b}}$ & 9,2 & 8,73 & & 15 & 10 & \\
\hline $\mathrm{G}_{\mathrm{a}}$ & 8,6 & 8,42 & \multirow[t]{2}{*}{8,41} & 75 & 50 & \multirow[t]{2}{*}{125} \\
\hline $\mathrm{G}_{\mathrm{b}}$ & 8,6 & 8,19 & & 15 & 10 & \\
\hline $\mathrm{G}_{\mathrm{a}}$ & 9,6 & 9,48 & \multirow[t]{4}{*}{9,53} & 75 & 5 & \multirow[t]{2}{*}{123} \\
\hline $\mathrm{G}_{\mathrm{b}}$ & 9,6 & 9,13 & & 15 & 1 & \\
\hline $\mathrm{G}_{\mathrm{a}}$ & 10,6 & 10,20 & & 75 & 1 & \multirow{2}{*}{122} \\
\hline $\mathrm{G}_{\mathrm{b}}$ & 10,6 & 9,90 & & 15 & 0,2 & \\
\hline$A_{a}$ & 4,75 & 4,44 & \multirow[t]{2}{*}{4,44} & 100 & 5 & \multirow[t]{2}{*}{166} \\
\hline$A_{b}$ & 4,75 & 4,27 & & 20 & 1 & \\
\hline $\mathrm{E} 5,8$ & & & & 50 & 5 & 148 \\
\hline $\mathrm{E} 6,8$ & & & & 50 & 25 & 158 \\
\hline E7, 8 & & & & 50 & 50 & 155 \\
\hline $\mathrm{E} 8,9$ & & & & 75 & 5 & 155 \\
\hline $\mathrm{E} 9,9$ & & & & 75 & 25 & 156 \\
\hline E10,9 & & & & 75 & 50 & 147 \\
\hline
\end{tabular}

Tableau 4. Composition des milieux d'insémination artificielle (expérience 6)

\begin{tabular}{cccc}
\hline $\begin{array}{c}\text { Nature de } \\
\begin{array}{c}\text { 'espèce tampon } \\
\text { et désignation } \\
\text { de la solution } \\
\text { mère }\end{array}\end{array}$ & $\begin{array}{c}\mathrm{pH} \\
\text { au } \\
\text { moment } \\
\text { de l'emploi }\end{array}$ & $\begin{array}{c}\text { Concentration } \\
\text { en espèce } \\
\text { chimique } \\
\text { tampon }([\mathrm{C}]) \\
(\text { mosmol } / \mathrm{kg})\end{array}$ & $\begin{array}{c}\text { Osmolarité } \\
(\mathrm{mosmol} / \mathrm{kg})\end{array}$ \\
\hline $\mathrm{M}-\mathrm{NaOH}$ & 10,4 & & \\
$\mathrm{M}$ & 10,2 & 100 & 150 \\
$\mathrm{G}-\mathrm{M}$ & 10 & & \\
$\mathrm{E}$ & 9,9 & 100 & 150 \\
$\mathrm{G}-\mathrm{M}$ & 10 & & \\
$\mathrm{G}$ & 9,6 & 100 & 150 \\
$\mathrm{~T}$ & 8,2 & 100 & 150 \\
$\mathrm{E}-\mathrm{HCl}$ & 7,0 & & 150 \\
$\mathrm{E}$ & 6,8 & 60 & \\
$\mathrm{M}-\mathrm{E}$ & 6,8 & & 150 \\
$\mathrm{M}-\mathrm{E}$ & 6,5 & & \\
$\mathrm{M}$ & 6,2 & 60 & \\
\hline
\end{tabular}

pH 10,2, EDA au pH 9,9, glycine au pH 9,5 et Tris au pH 8,2) et $60 \mathrm{mM} / \mathrm{kg}$ pour les DIA utilisant une deuxième charge ionique (EDA. au pH 6,8 et APM au pH 6,2).

$\Lambda$ côté de ces solutions utilisant les agents tampon au maximum de leur pouvoir tampon, des solutions de $\mathrm{pH}$ intermédiaires ont été préparées, soit en mélangeant deux à deux les solutions définies plus haut, soit en ajustant le pH par des solutions molaires. d'HCl ou de $\mathrm{NaOH}$ : il s'agit des DIA (notés M-E, tableau 4) préparés avec l'APM et l'EDA aux pH 6,5 et 6,6, du DIA noté E-HCl préparé avec l'EDA et
Tableau 5. Composition des milicux d'insémination artificielle (expérience 7)

\begin{tabular}{ccc}
\hline $\begin{array}{c}\text { Nature de } \\
\text { l'espèce tampon, et } \\
\text { désignation de la } \\
\text { solution mère et de } \\
\text { la série de dilution }\end{array}$ & $\begin{array}{c}\mathrm{pH} \\
\text { au moment } \\
\text { de l'emploi }\end{array}$ & $\begin{array}{c}\text { Concentration } \\
\text { en espèce chimique } \\
\text { tampon }([\mathrm{C}]) \\
\text { (mosmol } / \mathrm{kg})\end{array}$ \\
\hline $\mathrm{A}_{\mathrm{a}}$ & 5,0 & 100 \\
$\mathrm{~A}_{\mathrm{b}}$ & 5,0 & 33 \\
$\mathrm{M}_{\mathrm{a}}$ & 6,0 & 60 \\
$\mathrm{M}_{\mathrm{b}}$ & 6,0 & 20 \\
$\mathrm{E}_{\mathrm{a}}$ & 6,6 & 20 \\
$\mathrm{E}_{\mathrm{b}}$ & 6,6 & 20 \\
$\mathrm{E}_{\mathrm{a}}$ & 6,9 & 60 \\
$\mathrm{E}_{\mathrm{b}}$ & 6,9 & 20 \\
$\mathrm{~T}_{\mathrm{a}}$ & 8,2 & 100 \\
$\mathrm{~T}_{\mathrm{b}}$ & 8,2 & 33 \\
\hline
\end{tabular}

l'acide chlorhydrique, des DIA notés G-M préparés aux pH 9,8 et 10,2 avec la glycine et l'APM, et du DIA noté M-NaOH préparé au pH 10,4 à partir d'APM et de soude.

Expérience 7 (tableau 5). Acidotolérance dans un large volume de DIA à deux niveaux de pouvoir tampon à la limite de l'IpH-F

Comme dans l'expérience 6 des DIA, d'osmolarité 150 mosmol $/ \mathrm{kg}$, ont été préparés en placant les espèces tampon à des $\mathrm{pH}$ voisins de leur pK d'ionisation soit le pH 5 pour l'acide acétique, le $\mathrm{pH} 6$ pour l'APM, les pH 6,6 et 6,9 pour l'EDA et le $\mathrm{pH} 8,2$ pour le Tris. Dans une première série de tests (série A) les concentrations en 

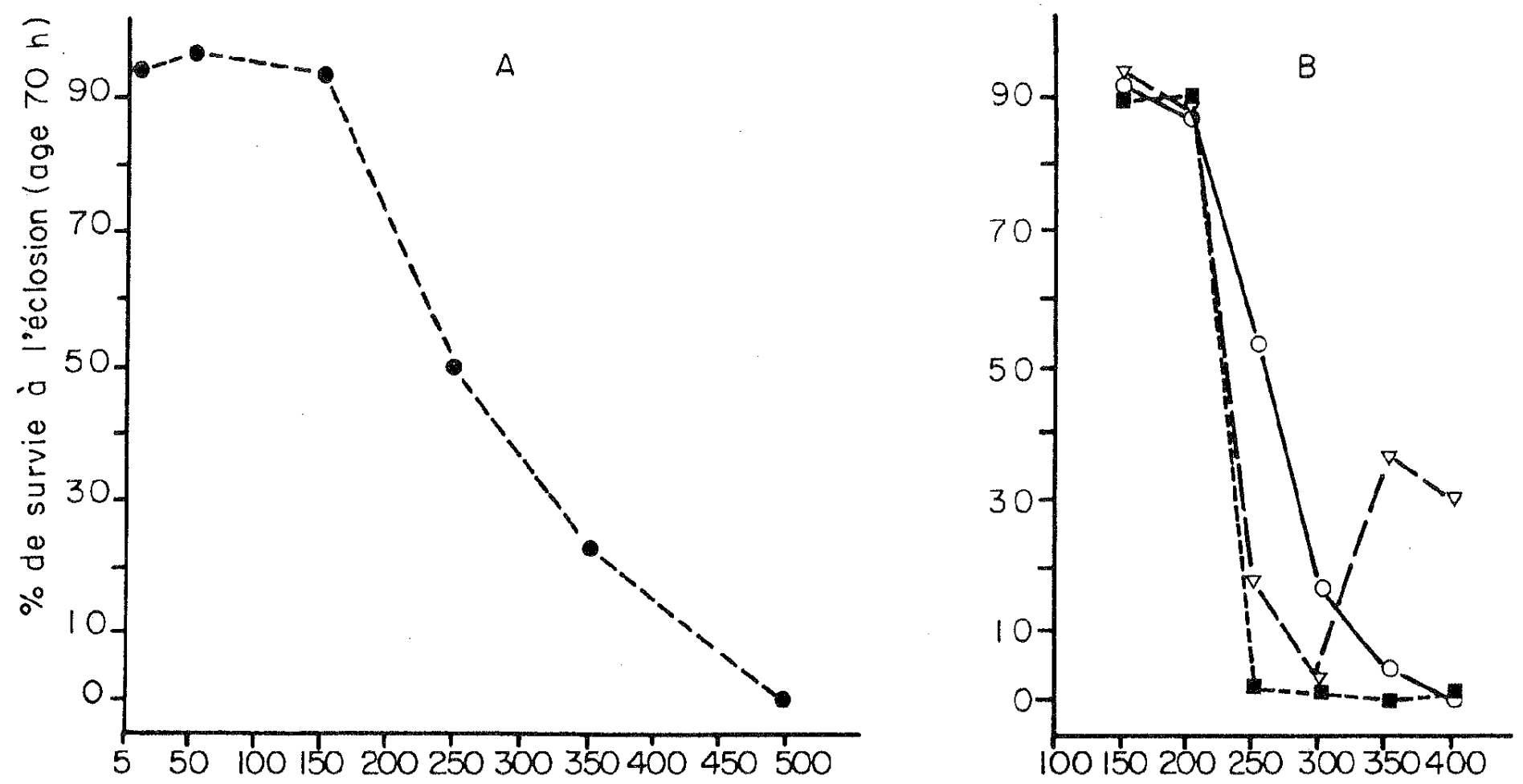

\section{Osmolarité du DIA (mosmol/kg)}

FIG. 1. Influence de l'osmolarité du DIA sur la survie embryonnaire avant éclosion (expérience 1). (A) DIA compósé de solution minérale non tamponnée (solution H). (B) DlA tamponné au pH 8,2 par du Tris-HCl (100 mosmol/kg) et complété par du $\mathrm{NaCl}(---)$, par du chlorure de chlorine $(---)$, ou par du glycérol (-).

espèce tampon s'élevaient à $100 \mathrm{~m} M / \mathrm{kg}$ pour le Tris et l'acide acétique, et à $60 \mathrm{~m} M / \mathrm{kg}$ pour l'EDA et l'APM. A partir des DIA de la série $\mathrm{A}$, les dilueurs de la série $\mathrm{B}$ ont été préparés par dilution au $1: 3$ par de l'eau du robinet.

A la différence des expériences précédentes, les oeufs ont été placés dans un large volume de DIA (200 $\mathrm{mL}$ par lot), ce qui a permis de supprimer la dérive de $\mathrm{pH}$ au cours d'expérience.

\section{Résultats}

Expérience 1 (fig. 1)

Avec une solution saline physiologique de $\mathrm{pH}$ non stabilisé (expérience 1A, fig. 1A), comme avec des solutions tamponnées de pH 8,2 (tampon Tris- $\mathrm{HCl}$ ), et contenant soit du $\mathrm{NaCl}$, soit du glycérol, soit du chlorure de choline (expérience 1B, fig. 1B), un effet négatif sur la survie embryonnaire n'apparait qu'au dessus d'une osmolarité de $200 \mathrm{mosmol} / \mathrm{kg}$.

\section{Expérience 2 (fig. 2)}

Dans un DIA concentré en agent chimique tampon et enrichi en sels minéraux (tableau 1 , série 2 , fig. $2 \mathrm{~A}$ ), la courbe de survie embryonnaire au moment de l'éclosion présente un maximum en plateau dans la gamme de $\mathrm{pH} 7,2$ et 9,2 , gamme qui appartient à ce que nous appelons, l'intervalle de $\mathrm{pH}$ favorable (IpH-F). Au delà de cet intervalle, soit pour les $\mathrm{pH} 6,2$ et 10,6 , la survie est nulle ou presque nulle. Au pH 7,2, le Tris donne des pourcentages de survie supérieurs à ceux fournis par le tampon phosphate.

Ces résultats restent inchangés, que l'exposition des oeufs à partir de l'insémination dure 30 (fig. 2A) ou 15 min (fig. 2B).

L'influence de la dilution des tampons se manifeste par une atténuation des effets aux pH extrèmes: $\mathrm{pH}$ 6,2 et 10,6, ainsi qu'au pH 7,2 avec le tampon phosphate. Le tableau 1 montre que, pendant les 30 min de séjour dans les boites de Petri durant la fécondation, le $\mathrm{pH}$ du DIA varie. Cette variation est d'autant plus importante que le DIA est plus dilué et que le $\mathrm{pH}$ est au début de l'expérience plus éloigné du $\mathrm{pH}$ voisin de 7,2 . Les $\mathrm{pH}$ semblent alors évoluer vers un $\mathrm{pH}$ limite voisin de pH $6,5-6,6$.

\section{Expérience 3 (fig. 3)}

Dans des DIA concentrés en agent chimique (45 et $50 \mathrm{~m} M / \mathrm{kg}$ ), mais préparés avec de l'eau bidistillée sans apport minéral (fig. 3), les résultats de l'expérience 2 sont pour l'essentiel retrouvés. Dans l'expérience 3B (fig. 3B), la borne supérieure de l'IpH-F est portée du côté alcalin jusqu'au pH 9,6. Au pH 7,2 et pour des comptages effectués au stade oeillé (âge de $48 \mathrm{~h}$ ), la survie est nulle avec le tampon phosphate et n'est que de $41 \%$ avec le tampon Tris. Avec le tampon acétate, cependant, la survie qui est nulle au $\mathrm{pH} 5$, est proche du maximum au $\mathrm{pH} 6,2$.

Entre le stade du bourgeon caudal (âge $30 \mathrm{~h}$ ) et le stade oeillé (âge $48 \mathrm{~h}$ ), le pourcentage de survie subit une chute importante au $\mathrm{pH} \mathrm{7,2} \mathrm{avec} \mathrm{le} \mathrm{Tris} \mathrm{et} \mathrm{le} \mathrm{phosphate} \mathrm{et} \mathrm{au} \mathrm{pH} \mathrm{10,6} \mathrm{avec} \mathrm{la}$ glycine.

\section{Expérience 4 (fig. 4)}

Une exposition préalable des ovules pendant $10 \mathrm{~s}$ avant l'insémination (courbe $a_{1}$ ) n'a pas d'effets différents sur la survie à l'éclosion de ceux produits par unc cxposition simultanée des ovules et du sperme (courbe $a_{2}$ ). Avec des DIA de concentration saline et osmolarité plus faibles que dans l'expérience 2 , les effets déjà constatés d'une dilution des DIA sont retrouvés. Avec les tampons à l'acide acćtiquc, les pourcentages de survie à l'eclosion restent nuls aux $\mathrm{pH} 4,75$ et 5,75, même lorsque les concentrations en espèces tampon sont relativement réduites: $[C]=20 \mathrm{mM} / \mathrm{kg}$. Avec le tampon phosphate concentré, $[C]=$ $37,5 \mathrm{mM} / \mathrm{kg}$, les pourcentages de survie à l'éclosion sont nuls aux $\mathrm{pH} 6,2,7,2$ et 8,2 (courbe $a$ ) alors q'une dilution au $1: 5$ 


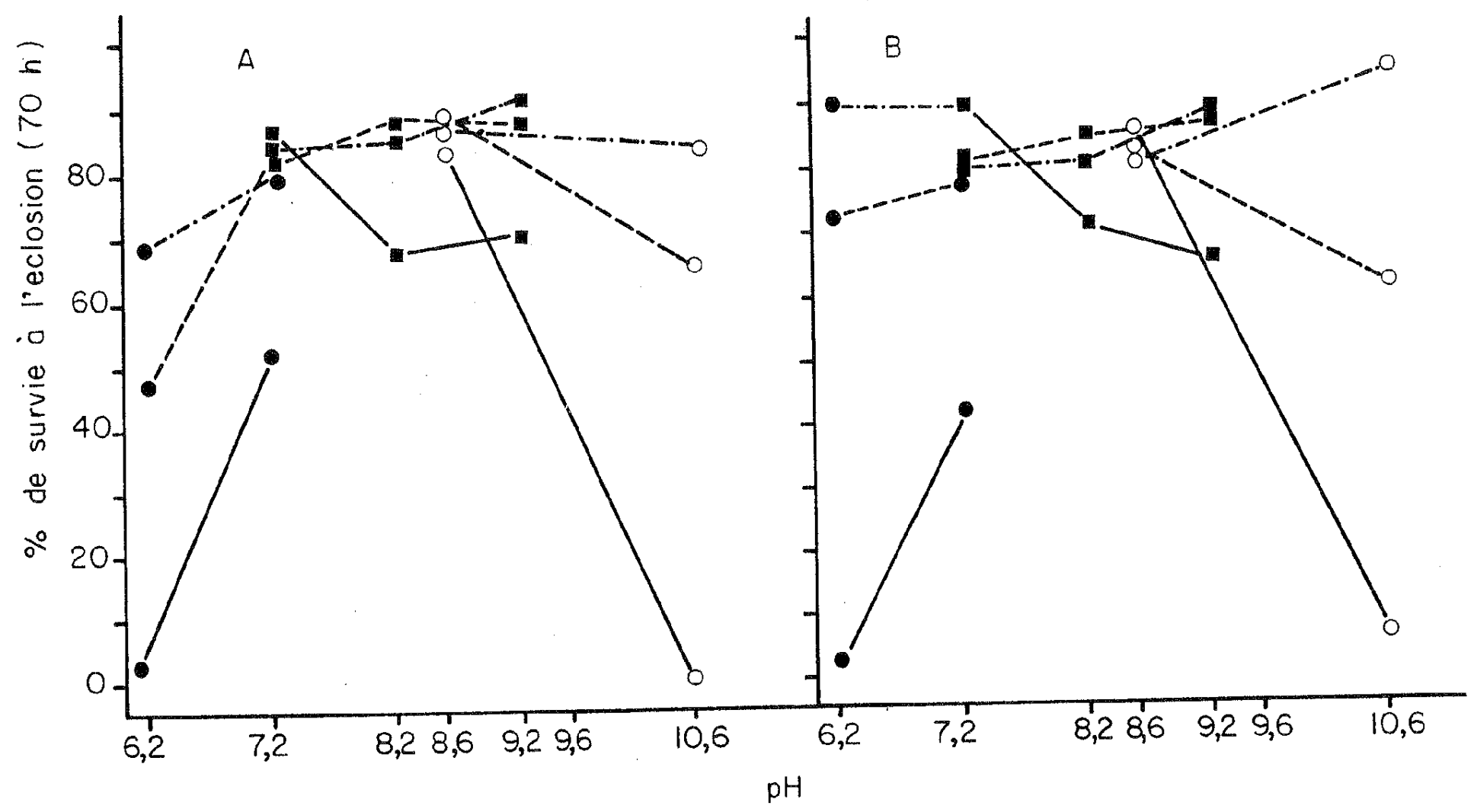

FIG 2. Survie embryonnaire au moment de l'éclosion en fonction du $\mathrm{pH}$, de la dilution d'un DIA enrichi en sels minéraux et de la durée du traitement (expérience 2). (A et $\mathrm{B}$ ) Séjour de 30 et 15 min dans le DIA à partir de l'insémination. DIA à l'acide phosphorique; $\mathbf{D}$, DIA au Tris: DIA à la glycine; - DIA concentré (série A d'osmolarité $150 \mathrm{mosmol} / \mathrm{kg}$ environ); - - - DIA dilué au $1 / 3$ (série B d'osmolarité $50 \mathrm{mosmol} / \mathrm{kg}$ environ); ·-.--, DIA dilué au $1 / 7,5$ (série C d'osmolarité $20 \mathrm{mosmol} / \mathrm{kg}$ environ).
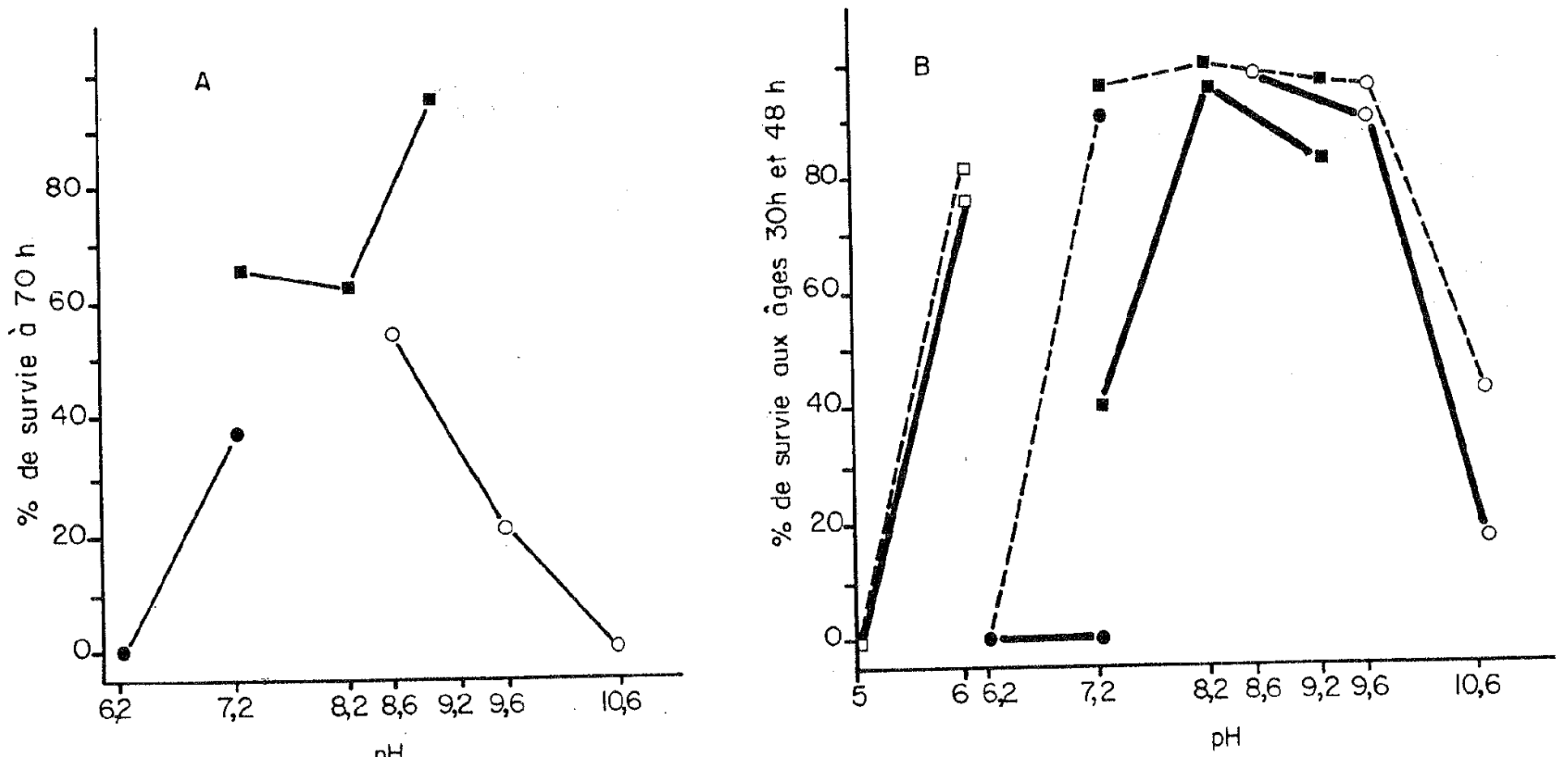

FIG. 3. Action de DIA concentrés, non minéralisés. Evolution de la survie au début de l'embryogénèse (expérience 3). (A) Résultats de l'expérience $3 \mathrm{~A}$. (B) Résultats de l'expérience 3B. $\square$, DIA à l'acide acétique; DIA à l'acide phosphorique; DIA au Tris; $O$, DIA à la glycine; - , \% de survie à l'éclosion (expérience 3A); ..., \% de survie au stade "bourgeon caudal" (âge $30 \mathrm{~h}$, expérience 3B); -, \% de survie au stade "oeillé" (âge $48 \mathrm{~h}$, expérience $3 \mathrm{~B}$ ).

atténue les effets au pH 6,2 et les supprime aux pH 7,2 et 8,2. La même influence de la dilution apparait au pH 9,6 avec le tampon glycine qui, à la concentration $[C]=75 \mathrm{mM} / \mathrm{kg}$, donne des pourcentages de survie à l'éclosion de $40 \%$ (courbes $a_{1}$ et $a_{2}$ ) et à la concentration $[C]=15 \mathrm{mM} / \mathrm{kg}$, donne un pourcentage de $79 \%$.

Une dérive de $\mathrm{pH}$ (tableau 2) au cours du traitement est observée; elle est comparable à celle observée dans l'expé- 


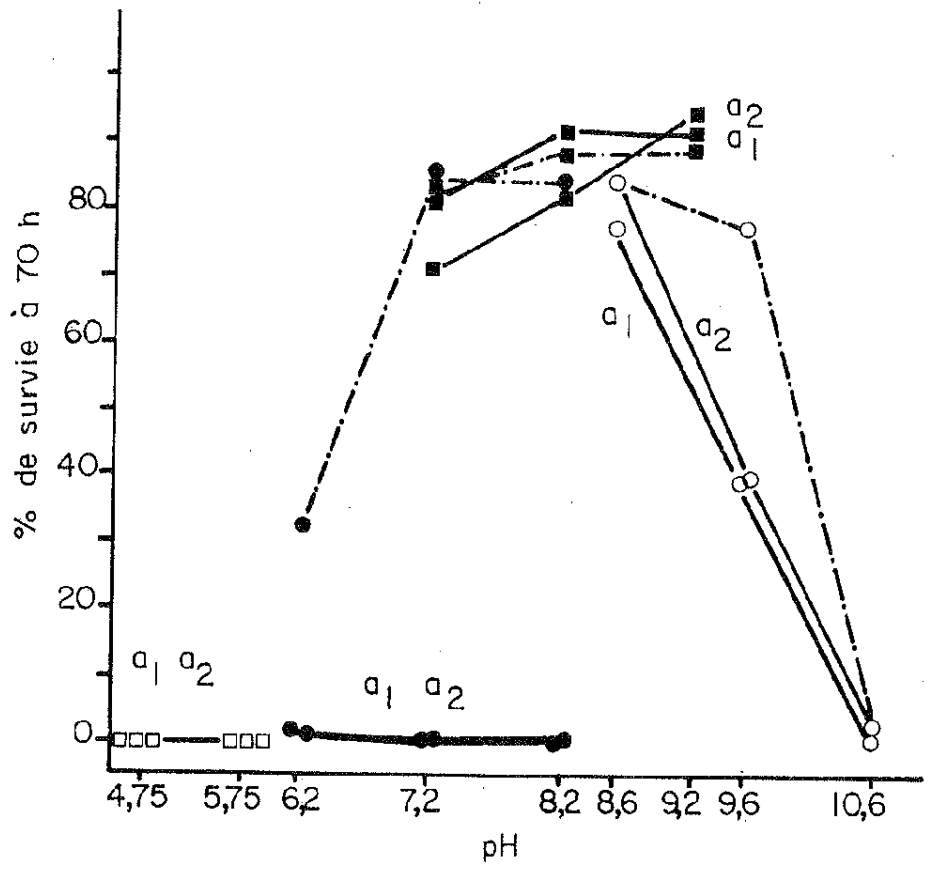

FIG. 4. Détermination de l'IpH-F à deux concentrations de DIA. Exposition des ovules avant insémination et exposition simultanée des gamètes au moment de l'insémination (expérience 4, tableau 3). - , DIA concentrć; - - - - D DIA dilué au $1 / 5 ; a_{1}$, exposition des ovules $10 \mathrm{~s}$ avant l'introduction du sperme; $a_{2}$, introduction simultanée des ovules et du sperme; $\square$, DIA à l'acide acétique; DIA à l'acide phosphorique; DIA au Tris; $O$, DIA à la glycine.

rience 1 (tableau 1), le point d'équilibre étant le $\mathrm{pH}$ 6,1, sauf avec l'acide acétique où il semble inférieur à 4 .

\section{Expérience 5 (fig. 5)}

L'expérience 5 (tableau 3) permet de comparer ces deux femelles différentes, les effets de DIA concentrés et de tester les effets de l'EDA. Les deux femelles présentent des tolérances comparables avec les ions glycine, Tris, phosphate et acétate, mais différentes vis à vis de l'EDA. D'autre part, avec cet ion, l'IpH-F paraît s'étendre du côté acide jusqu'au pH 6,8 et il existe encore des survivants à l'éclosion avec le pH 5,8 , tandis qu'un pH de 5,75 est lćthal pour tous les oeufs avec 1 'acide acétique. Les effets de l'ion phosphate sont plus importants aux pH 6,2 et 8,2 qu'au pH 7,2. La borne supérieure de l'IpH-F est située entre le pH 9,6 (tampon à la glycine) et le $\mathrm{pH}$ 9,9 (tampon à l'EDA).

\section{Expérience 6 (fig. 6, tableau 4)}

Du côté des $\mathrm{pH}$ alcalins, la chute des pourcentages de survic des embryons pré-éclos est complète en 0,3 unité de $\mathrm{pH}$, entro les valeurs 9,6 et 9,9. Du côté des pH acides, cette chute de la survie embryonnaire est plus progressive; elle a déjà commencé au pH 7, où l'on ne trouve plus que $59 \%$ de survivants (contre $89 \%$ au pH 8,2 avec le Tris) et elle n'est pas achevée au $\mathrm{pH} 6,5$ où il reste encore $5 \%$ de survivants avec un DIA à l'APM-EDA.

\section{Expérience 7 (fig. 7, tableau 5)}

Avec des DIA concentrés et présentant un fort pouvoir tampon, la chute de tolćrance est brutale au voisinage de la neutralité puisqu'au pH 6,9 , on ne retrouve plus que $35 \%$ de survivants à l'éclosion. Cependant, une dilution du DIA au $1: 3$ atténue cet effet, puisqu'il reste encore $64 \%$ de survivants au pH 6,9 et $53 \%$ au pH 6, mais la dilution ne semble pas capable de déplacer sensiblement la borne inférieure de l'IpH-F.

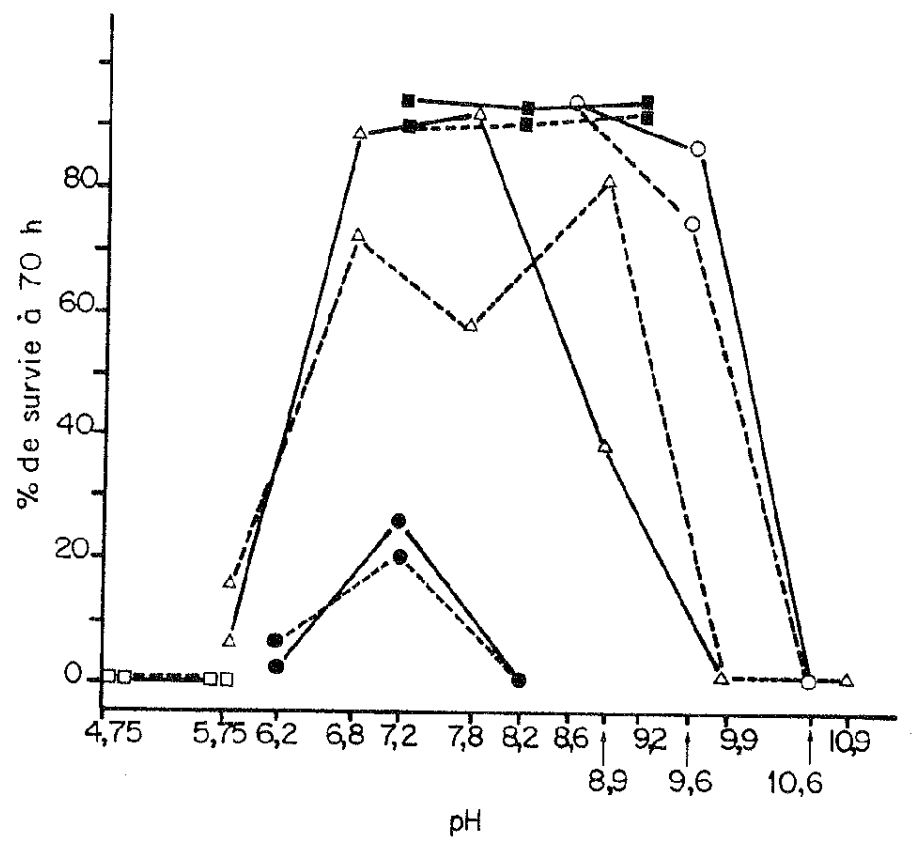

FIG. 5. Détermination de l'IpH-F sur les ovules séparés de deux femelles essais de l'ethylènediamine (EDA) comme agent tampon (expérience 5, tableau 3). - , résultats obtenus avec les ovulcs de la femelle $A$; ---, résultats obtenus avec les ovules de la femelle $B$; $\square$, DIA à l'acide acétique; DIA à l'acide hosphorique; $\mathbf{D}$, DIA au Tris; $O$, DIA à la glycine, $\triangle$, DIA à l'EDA.

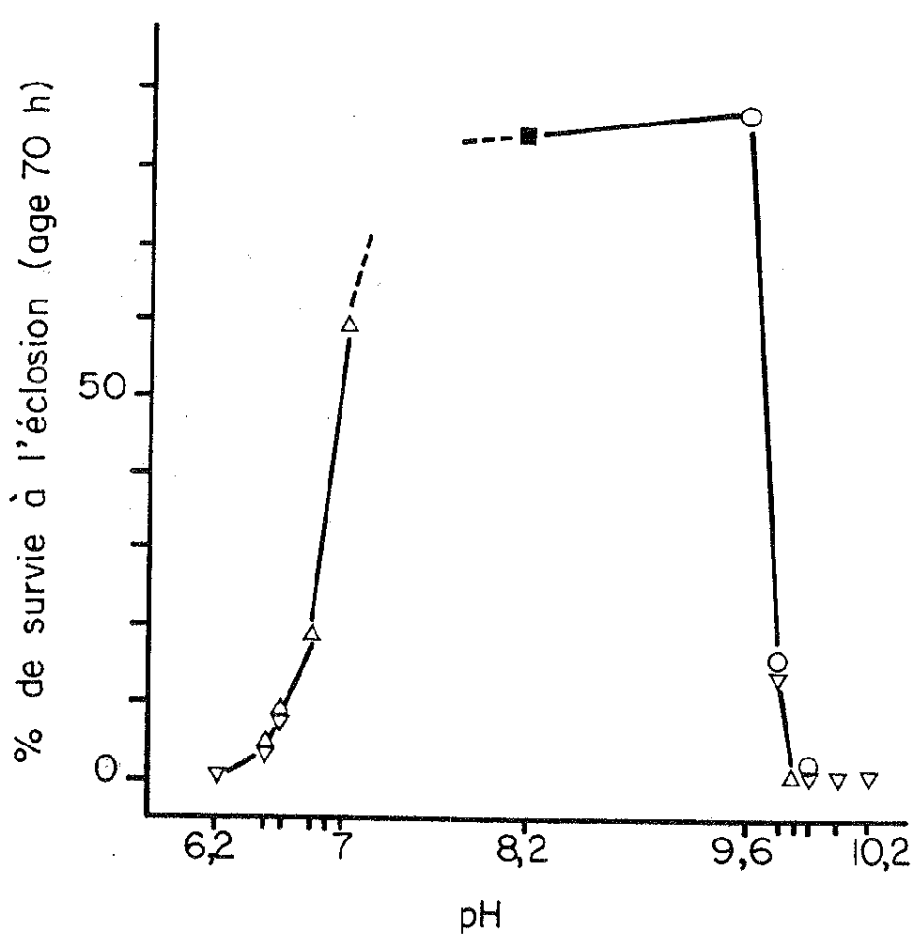

FIG. 6. Résistance de l'oeuf aux bornes de l'IpH-F (expérience 6, tableau 4) essai de l'aminopropylmorpholine (APM) comme agent tampon. $\nabla$, DIA à l'APM; $\theta$, DIA à l'APM et à l'EDA; $\triangle$, DIA à I'EDA; 1 , DIA au Tris; $O$, DIA à la glycine; $\theta$, DIA à la glycine et à !'APM

\section{Discussion}

Dans toutes nos expériences sur les effets de $\mathrm{pH}$, et particulièrement avec des DIA concentrés, il n'est pas possible de distinguer complètement l'action propre du $\mathrm{pH}$ de celle qui résulte d'une toxicité spécifique des molécules et ions utilisés comme agent tampon. En effet, l'action du pH sur les proces- 


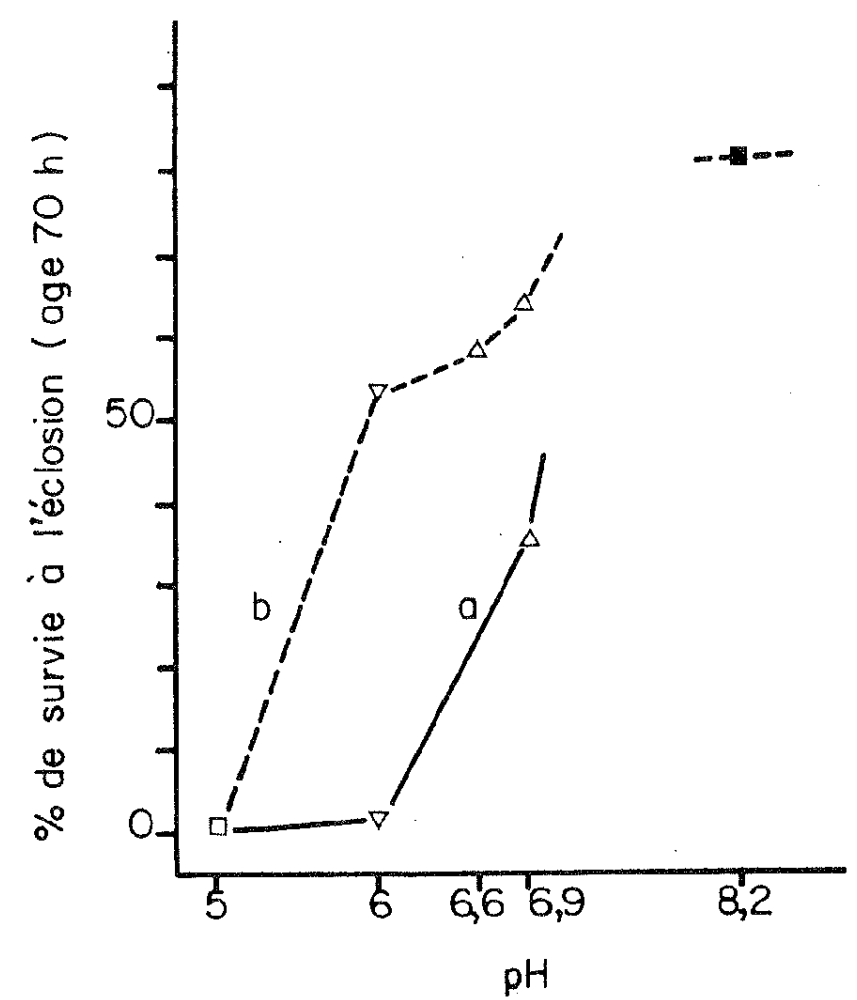

Fig. 7. Acidorésistance et effet de dilution à la limite de l'IpH-F. - DIA concentré; -.-, DIA dilué au $1 / 3 ; \square$, DIA à l'acide acétique; $\nabla$, DIA à l'APM; $\triangle$, DIA à l'EDA.

sus complexes qui se succèdent au cours de la fécondation dépend directement de la capacité des agents tampon à diffuser au contact des gamètres et à pénétrer les barrières biologiques que constituent la membrane du spermatozoïde, la coque de l'oeuf, l'espace périvitellin en formation, la membrane plasmique périvitelline et le cytoplasme de l'oeuf en remaniements accélérés.

\section{Durée du traitement}

Les expériences $2 \mathrm{~A}$ et $2 \mathrm{~B}$ qui comparent les effets d'un séjour de 15 et 30 min dans le DIA indiquent que l'essentiel des effets de pH est déjà obtenu dans les 15 premières minutes du développement. Mais ceci n'indique nullement que l'oeuf soit devenu tolérant après cette période car nous avons observé par ailleurs que la sensibilité à des chocs acides se maintient pendant plusieurs heurcs après l'insémination.

\section{Dispersion des spermatozoïdes}

L'expérience 4, de son côté, montre qu'une exposition des ovules pendant $10 \mathrm{~s}$ avant l'insémination, ne suffit pas pour modifier sensiblement leur fécondabilité. Si cette observation permet d'exclure un effet a priori possible de légers retards expérimentaux dans l'introduction du sperme sur les ovules, elle n'autorise pas à faire du spermatozoïde libre la cible privilégiée de l'action du $\mathrm{pH}$. Des expériences en cours semblent d'ailleurs nous montrer, avec des DIA concentrés, qu'unc exposition des spermatozoüdes pendant quelques dizaines dc secondes avant insémination, ne modifie pas sensiblement les profils de tolérance aux effets de $\mathrm{pH}$.

La détermination de l'IpH-F et celle de l'intervalle limite de tolérance (IpH-L) au pH supposent que les effets de l'osmolarité et de la composition minérale des DIA ne puisse interférer avec les effets spécifiques du pH.

\section{Pression osmotique}

Les résultats de l'expérience 1 montrent que la concentration osmotique du DIA ne devient un factcur déterminant pour la survie embryonnaire à l'éclosion, qu'au dessus de la valeur $200 \mathrm{mosmol} / \mathrm{kg}$; ceci démontre que nos expériences sont restées dans le domaine acceptable quant à ce paramètre.

\section{Composition minérale}

Les résultats des cxpériences $3,4,6$ et 7 montrent aussi que les différentes compositions minérales utilisées pour la fabrication des DIA n'ont pas interféré avec le facteur $\mathrm{pH}$.

\section{Effets spécifiques des agents tampon}

La courbe de survie obtenue dans l'expérience 6 donne une idée assez précise de l'effet spécifique du pH sur la fécondation. L'IpH-F s'étend d'un pH légèrement supérieur à la neutralité jusqu' au pH 9,6. Au delà de ces valeurs, la tolérance de l'oeuf de carpe chute brutalement du côté alcalin et plus progressivement du côté acide, déterminant pour l'IpH-L les valeurs $\mathrm{pH}$ 6,2-9,9. Ce résultat n'est selon nous contredit qu'en apparence par des observations partielles de plusieurs expériences.

Dans l'expérience 3, on obtient au pH 6 un pourcentage de survie important (77\%) avec l'acide acétique comme tampon (fig. 3A). Mais on peut interpréter ce résultat en remarquant qu'à un pH aussi ćloigné du pK d'ionisation de l'acide acétique $(\mathrm{p} K=4,75)$, le pouvoir tampon de cet agent est négligeable.

Avec l'éthylc̀ne diamine, l'expérience 5 donne, au $\mathrm{pH} \mathrm{6,8}$, des pourcentages de survie plus importants que ceux obtenus dans l'expérience 6 , bien qu'inégaux suivant la femelle utilisée. Il est possible que la concentration en tampon légèrement différente $([C]=50 \mathrm{mM}$ dans l'expérience 5 et $[C]=60 \mathrm{mM}$ dans l'expérience 6; tableau 4) explique ces variations apparentes de la borne acide de l'IpH-F. Il peut exister aussi des différences liées aux caractéristiques des géniteurs. Enfin, une cvolution différente du pH du DIA en cours d'expérience peut aussi être invoquée. Par ailleurs, les pourcentages non nuls de survie obtenus au pH 5,75 dans l'expérience 5 peuvent s'expliquer par la faiblesse du pouvoir tampon de l'EDA à ce pH puisque le $\mathrm{p} K$ d'ionisation le plus proche est de $\mathrm{pH} 6,8$.

Du côté alcalin, les pourcentages de survie non nuls obtenus au $\mathrm{pH} 10,6$ dans l'expérience $3 \mathrm{~B}$, s'expliquent par le caractère différé de l'effet du pH sur la mortalité. Les comptages ont été effectués à 30 et $48 \mathrm{~h}$, c'est-à-dire nettement avant l'éclosion (72 h)

Le pH 9,6 n'est pas toujours inclu dans l'IpH-F (expériences $3 \mathrm{~A}$ et 4 , tampon glycine). Ceci peut s'expliquer si le $\mathrm{pH} 9,6$ représente la borne extreme de l'IpH-F. Par contre, la valcur basse du pourcentage de survie obtenu au $\mathrm{pH} 8,9$ pour la femelle A dans l'expérience 5 ne se retrouve pas pour l'autre femelle (B), ce qui semble indiquer une variabilité interfemelle dans la qualité des ovules et leur tolérance aux $\mathrm{pH}$ alcalins lors de la fécondation.

Les résultats obtenus avec l'APM sont cohérents avec ceux fournis par les autres tampons.

\section{Toxicité des ions phosphate}

Les DIA contenant de l'acide phosphorique sont toxiques. Au pH 8,2, le pouvoir tampon de l'acide phosphorique est relativement faible (puisque le $\mathrm{p} K$ vaut 7,2 ) et partout, cependant, les pourcentages de survie sont nuls (sauf dans l'expérience $4 \mathrm{~B}$ dans laquelle la concentration $([C]=7,5 \mathrm{mM})$ est probablement trop faible pour que l'agent tampon ait un effet notable)

Au pH 6,2, par contre, l'acide phosphorique ne parait pas 
faire exception car le maintien des pourcentages de survie non nuls (bien que faibles) dans les expériences 4 et 5 peut s'expliquer par un pouvoir tampon beaucoup plus faible que celui de l'APM au même $\mathrm{p} K$.

Au pH 7,2 enfin, les pourcentages de survie sont toujours plus faibles avec l'acide phosphorique qu'avec le Tris (expériences $2 \mathrm{~A}, 2 \mathrm{~B}, 3 \mathrm{~A}, 3 \mathrm{~B}, 4,5 \mathrm{~A}$ et $5 \mathrm{~B}$ ); cette différence pourrait être due en partie aux différences de pouvoir tampon entre ces deux agents à ce $\mathrm{pH}$ (l'acide phosphorique étant à son $\mathrm{pK}$ d'ionisation, le Tris à une unité de $\mathrm{pH}$ de ce $\mathrm{p} K$ ). Cependant, l'effet du pouvoir tampon ne peut être invoqué dans l'expérience 5 pour la comparaison de l'acide phosphorique avec l'EDA. Ce dernier agent tampon est utilisé à son $\mathrm{p} K$ d'ionisation qui est plus bas que celui de l'acide phosphorique. Or, avec des concentrations de $50 \mathrm{mM}$, l'EDA au pH 6,8 donne des pourcentages de survie plus élevés que l'acide phosphorique à la concentration de $37,5 \mathrm{mM}$ et au $\mathrm{pH} 7,2$. Il existe donc une toxicité particulière de l'acide phosphorique, décelable au $\mathrm{pH}$ 7,2 et très forte au $\mathrm{pH} 8,2$.

La mise en évidence de cette toxicité pourrait remettre en question certains résultats obtenus à l'aide de milieux tamponnés au phosphate quant à la physiologie de l'activation (Cykowska et Winnicki 1972; Winnicki et Cykowska 1973) ou quant à la définition des $\mathrm{pH}$ favorables à l'insémination artificielle (Petit et al. 1973; Billard 1977). Nous émettons l'hypothèse que la toxicité des phosphates pourrait être liée au rôle de ces ions dans la précipitation du calcium. En effet, le pouvoir précipitant du tampon à l'acide phosphorique est d'autant plus important que le $\mathrm{pH}$ est plus élevé puisqu'il dépend de la concentration en ions $\mathrm{PO}_{4}^{3-}$. D'autre part, Gilkey (1981) démontre le rôle déterminant du calcium dans le déclenchement de tous les processus de la fécondation: le $\mathrm{pH}$ du milieu agirait dès lors en modifiant le seuil de concentration calcique nécessaire à l'activation. Brown et Lynan (1981) ont montré que la concentration en ions calcium dans le milieu exerce, entre 0 et $10 \mathrm{mg} / \mathrm{L}$, une action déterminante sur la survie embryonnairc et cette action est d'autant plus importante que le $\mathrm{pH}$ est plus bas. Dans nos expériences, d'ailleurs, celles qui mettent en oeuvre des DIA préparés à l'eau déionisée (expériences $3 \mathrm{~A}, 3 \mathrm{~B}$ ) fournissent, même avec le Tris, des pourcentages de survie particulièrement faibles. Cependant, au moment de l'introduction des ovules et du sperme, une certaine quantité de calcium est apportée dans le milieu. On peut penser que le $\mathrm{pH}$ du milieu agit alors en influençant l'évolution des équilibres entre le calcium cytoplasmique de l'oeuf, le calcium retenu dans l'espace périvitellin en formation et dans la zona radiata et le calcium du milieu.

Les grosses moléculcs mucoprotéiniques qui sont maintenues dans l'espace périvitcllin par la barrière de la coque sont peut-être capables de retenir le calcium; cette hypothèse converge avec celle qui attribue au liquide pćrivitellin une fonction dans la réassociation post-mitotique des blastomères (Roubaud et Pairault 1980). Elle est cohérente avec l'observation de A. A. Neyfack (communication personnellc) sclon laquelle l'oeuf privé de sa coque ne se développe pas dans l'cau douce, mais se développe un certain temps dans une solution physiologique contenant du calcium.

\section{Nécessité des pH alcalins, effets de la dilution du DIA}

L'alcalinité marquée de l'intervalle de pH favorable et la dépendance de cet intervalle par rapport au pouvoir tampon du milieu caractérisent les besoins de la carpe commune au moment de la fécondation.

\section{Recherches écologiques}

La plupart des connaissances sur les effets de $\mathrm{pH}$ au cours du développement embryonnaire des poissons concerne des caux acidifiées par l'acide sulfurique. C'est, en effet, cet acide qui est le facteur principal des pollutions acidifiantes du milieu aquatique (Anonyme 1980). Cet agent étant un acide fort, les tests de résistance qui s'y rapportent utilisent, en large volume, un milieu non tamponné, et évitont les évolutions de pII en cours d'expérience soit en utilisant dc larges volumes, soit en renouvelant le milieu d'incubation. Ce type d'expérimentation est aussi utilisé lorsque l'agent acidifiant choisi est l'acide chlorhydrique (Kiyashko et Volodin 1978) qui, comme $\mathrm{SO}_{4} \mathrm{H}_{2}$, n'exerce aucun effet tampon. Presque toutes les recherches utilisant ce modèle expérimental, que nous qualificrons "d'écologique" (Beamich et Harvey 1972; Menendez 1976; Craig et Baksi 1977; Trojnar 1977a, 1977b; Daye et Garside 1977, 1979; Carrick 1979; Gjedrem 1980a, 1980b; Lec ct Gcrking 1980; Peterson et al. 1980; Brown 1981), déterminent l'acidorésistance des oeufs au cours de l'embryogénèsc mais seulement après la fécondation (à l'exception cependant de Craig et Baksi (1977) et Carrick (1979)). Cependant, dans ce cas, le milieu d'accompagnement des gamètes (liquide séminal, liquide ovarien ou coelomique) ayant un $\mathrm{pH}$ ćlevé (Baynes et al. 1981; Plouidy et Billard 1982), il est pratiquement impossible de réguler le $\mathrm{pH}$ au cours des premiers instants de l'insémination sans avoir recours à des agents tampons (Baynes et al. 1981). L'utilisation d'un très large volume utile, ou le renouvellement rapide du milieu, entre, en effet, en contradiction avec la nécessité de maintenir une concentration suffisante en spermatozoïdes autour des ovules.

\section{Recherches physiologiques}

Les études qui se sont données pour objet d'améliorer les techniques de l'insémination artificielle aboutissent à un autre modèle expérimental, que nous qualifierons de "physiologique," puisque l'objectif est de déterminer la composition d'un dilueur d'insémination artificielle (DIA) stable et de maintenir le $\mathrm{pH}$ optimum pendant la fécondation (Petit et al. 1973; Billard 1977; Marcel 1981). Dans ce modèle physiologique, c'est aux pH nettement alcalins que l'on aboutit, à des concentrations élevées en espèce tampon; $\mathrm{pH} \mathrm{9,2} \mathrm{et} \mathrm{concentration} \mathrm{en}$ espèce tampon de $70 \mathrm{~m} M$ pour le DIA 532 de truite (Billard 1977). Les acides utilisés (grâce à leur concentration) traversent rapidement la barrière de la coque de l'ovule ou de l'oeuf, et peut-être aussi celle de la membrane plasmique. Au contraire, dans le modèle expérimental "écologique", la zona radiata et l'espace périvitellin qui se forme pendant la fécondation opposent une résistance aux variations externes du $\mathrm{pH}$. Par exemple, pour le saumon atlantique, il faut environ 8 h pour que le $\mathrm{pH}$ du liquide périvitellin s'équilibre avec un milieu non tamponné de pH 5,5, et, avec un milieu de $\mathrm{pH} 4$, le $\mathrm{pH}$ du liquide périvitellin se maintient encore à la valeur de 4,2524 h après la baisse du pH externe (Peterson et al. 1980). Il est cependant probable que les vitesses d'équilibration du $\mathrm{pH}$ périvitellin dépendent, en même temps, de la composition ionique de l'eau, de sa vitesse d'écoulement autour de l'oeuf

De toutes façons, dans les conditions naturelles, le pouvoir fécondant du spermatozoïde ne se maintient, suivant les espèces, que quelques dizaines de secondes à quelques minutes après émission dans l'eau (Billard 1977; Baynes et al. 1981; P. Roubaud, Ch. Gillet et R. Billard, travail en cours). Par conséquent, les pH effectifs du milieu local dans lequel se déroule la fécondation, ainsi que les toutes premières minutes 
du développement, sont largement déterminés par les pH et pouvoirs tampon des liquides séminaux ou périovulaires, en même temps que par la vitesse de leur dispersion dans le milieu.

Avec des espèces comme la carpe, qui pond sur un substrat végétal dans des eaux calmes et de faible profondeur (Soin 1977), et qui libère des volumes importants d'ovules enrobés dans un liquide ovarien visqueux et nettement alcalins (Plouidy 1982), le pH réel qui présidc à la fécondation est peut-être relativement indépendant du milieu, des lors que celui-ci n'est pas tamponné. Cepcndant, comme l'indiquent nos expériences, et en particulicr l'expérience 7 (fig. 7), la dilution de l'agent tampon dans le DIA se traduit plus par une diminution dc la pente de la courbe d'acidorésistance que par l'abaissement du pH limite de l'intervalle favorable. Autrement dit, les pH situés en dessous de ce $\mathrm{pH}$ limite, qui correspond à peu près à la ncutralité, exercent toujours un effet défavorable sur la survic, et c'est seulement l'ampleur de cet effet qui parait déterminé par le pouvoir tampon du milieu. Cet effet négatif de l'acidité du milieu dès le seuil de neutralité, est.sans doute plus important dans les conditions naturelles, que ne le laissent paraître nos résultats. En effet, nous avons vérifié par ailleurs que l'embryon reste particulièrement sensible à l'effet de $\mathrm{pH}$ pendant quelques dizaines de minutes après les 15 ou 30 min qu'ont duré les expositions dans nos expériences. En outre, nous n'avons, dans celle-ci, comptabilisé que les mortalités avant éclosion. Or l'expérience 3 démontre que des mauvaises conditions de $\mathrm{pH}$ au moment de la fécondation exercent des effets retardés sur la survie embryonnaire. Cette observation vient soutenir une idée, de portée plus générale, de l'existence dans le cycle vital des organismes de relations différées (Jaoul et Roubaud 1982); on sait par exemple, que des chocs thermiques survenant au tout début du développement embryonnaire, peuvent n'affecter les pourcentages de survie qu'à la fin du développement précoce, et même ne se manifester qu'au delà de l'éclosion (Gervai et al. 1980). Il a aussi été montré (Gilkey 1981) que les premières secondes qui suivent la pénétration dı spermatozoüde sont décisives, non pas pour la suite immédiate du développemnt embryonnaire, mais pour des stades plus avancés: à partir de la gastrulation et au delà. Il est donc possible que les effets de bas $\mathrm{pH}$ ou des $\mathrm{pH}$ alcalins dans le milieu de fécondation puissent se traduire par des mortalités au delà de l'éclosion. Nos expériences démontrent l'importance du pouvoir tampon du milieu pour l'évaluation des effets de $\mathrm{pH}$ au moment de la fécondation. Ces effets dépendent de la vitesse de mise en équilibre des gamètes et de l'oeuf ayec le milieu. Il ne semble donc pas possible de déterminer les limites écologiques de $\mathrm{pH}$, sans une étude détaillée des conditions comportementales et hydrodynamiques qui président à cette mise en équilibre au moment de la fraie.

\section{Remerciements}

Nous remercions le Dr. A. A. Neyfack qui nous a communiqué d'intéressantes observations personnelles, ainsi que les personnes suivantes pour leurs collaborations scientifiques et matérielle: pisciculture, D. Marie, P. Le Brenn et M. Levilain; comptage et mesures, C. Ledez et P. Reinaud; bibliographie, B. Chevassus, M. H. Magri, J. Marcel et D. Marie; traduction, N. Zuzine et A. Daifuku; dosage biologique de la $c-G T H$, B. Jalabert. Ce travail a bénéficié du soutien du Secretariat d'Etat à l'Environnement (contrat 83.40.170) et du Centre National de la Recherches Scientifiques (R.C.P. L8 06 56).
ANONYME. 1980. Ecological impact of acid precipitation. Proceedings of the International Conference on the Ecological Impact of Acid Precipitation. SNSF Project, Åas, Norway.

BAynes, S. M., A. P. SCOTT, et A. P. DAwson. 1981. Rainbow trout, Salmo gairdneri Richardson, spermatozoa: effects of cations and pH on motility. J. Fish Biol. 19: 259-267.

BEAMISH, R. J, et H. H. HARVEY. 1972. Acidification of the La Cloche Mountain Lakes, Ontario, and resulting fish mortalities. J. Fish. Res. Board Can. 29: 1131-1143.

BILLARD, R. 1977. Utilisation d'un système Tris-Glycocolle pour tamponner le dilueur d'insémination de Truite. Bull. Fr. Piscic. 264: $102-112$.

BILlARD, R., et P. ROUBAUD. 1984. The effects of metals and cyanide ions on gamete survival and fertilization in rainbow trout (Salmo gairdneri). Water Res. In press.

BRown, D. J. A. 1981. The effects of various cations on the survival of brown trout, Salmo trutta, at low pHs. J. Fish Biol. 18: 31-40.

BRown, D. J. A., et S. LYNAN. 1981. The effects of sodium and calcium concentrations on the hatching of eggs and the survival of yolk sac fry of brown trout, Salmo trutta L., at low pH. J. Fish Biol. 19: $205-211$

CARRICK, T. R. 1979. The effect of acid water on the hatching of salmonid eggs, J. Fish Biol. 14: 165-172.

CraIG, G. R., et W. J. BAKSI. 1977. The effects of depressed $\mathrm{pH}$ on flagfish reproduction growth and survival. Water Res. 11: $621-626$

CykowskA, C, et A. WINNICKI. 1972. Embryonic development of the baltic sea trout, Salmo trutta L, in a buffer solution. Acta Ichthyol. Piscatoria, 11: 3-13.

DAYE, P, G., et E. T. GARSIDE. 1977. Lower lethal levels of $\mathrm{pH}$ for embryos and alevins of Atlantic salmon, Salmo salar L. Can. J. Zool. 55: $1504-1508$.

1979. Structural alterations in embryos and alevins of the Atlantic salmon, Salmo salar L., induced by continuous or shortterm exposure to acidic levels of pH. Can. J. Zool. 57: 1713-1718.

Gervai, J., S. Peter, A. Nagy, L. Horvath, et V. CSanyl. 1980. Induced triploidy in carp (Cyprinus carpio L.). J. Fish Biol. 17: $667-671$

GILlet, C, et P. Roubaud. 1983. Influence sur la survic jusqu'à l'éclosion des embryons de carpe commune (Cyprinus carpio L.) après traitement, pendant la fécondation et le développement précoce, par le carbendazime, un fongicide antimitotique de synthèse. Water Res. 17: 1343-1348.

GILKEY, C. 1981. Mechanisms of fertilisation in fishes. Am. Zool 21: $359-375$

GJEDREM, T, 1980a. Genetic variation in acid tolerance in brown trout. Proceedings of the International Conference on the Ecological Impact of Acid Precipitation. SNSF Project. Áas, Norway. p. 308.

$1980 \mathrm{~b}$. Growth and survival of fingerlings in acidic water. Proceedings of the International Conference on the Ecological Impact of Acid Precipitation. SNSF Project, Ảas, Norway. p. 309.

JALABERT, B., B. BRETON, et R. BILLARD. 1974. Dosage biologique des hormones gonadotropes de poissons par le test de maturation in vitro des ovocytes de truite. Ann. Biol. Anim. Biochem. Biophys. 14: 217-228.

JaOUl, A., et P. Rovbaud. 1982. Résistance de l'oeuf de carpe commune (Cyprinus carpio L. Cyprinidae) à des chocs thermiques chauds ou froids. Can. J. Zool. 60: 3409-3419.

Johansson, N., et G. Milbrink. 1976. Some effects of acidified water on the early devclopment of roach (Rutilus rutilus) and perch (Perca fluviatilis L.) Water Resour. Bull. 12: 39-48.

KIYASHKO, V. I., et V. M. VOLODIN. 1978. The influence of the embryonic development of the ruffe, Acerina cernua, of the active response of environment. J, Ichthyol. (Engl. Transl.), 18: $693-695$.

LeE, R. M., et S. D. Gerking. 1980. Sensitivity of fish eggs to acid stress. Water Res. 14: 1679-1681.

MARCEL, J. 1981. Contrôle de la reproduction et gestion des gamètes de quelques espèces de poissons téléostéens. Diplôme, École 
Pratique des Haute Études

MCKIM, M. C. 1977. Evaluation of tests with early stages of fish for predicting long term toxicity, J. Fish. Res. Board Can. 34: $1148-1154$

MENENDEZ, R, 1976. Chronic effects of reduced $\mathrm{pH}$ on brook trout (Salvelinus fontinalis). J. Fish. Res. Board Can. 34: 118-123.

Petit, J, B. Jalabert, B. Chevassus, et R. Billard. 1973. L'insémination artificielle de la truite (Salmo gairdneri Richardson). I. Effects du taux de dilution, du $\mathrm{pH}$ et de la pression osmotique du dilueur sur la fécondation. Ann. Hydrobiol. 4: 201-210.

Peterson, R. H., P. E. Daye, et J. L. MetCalfe. 1980. Inhibition of Atlantic salmon (Salmo salar). Hatching at low pH. Can. Fish. Aquat. Sci. 37: 770-774.

PLouIDY, M. G. 1982. Composition chimique des liquides d'accompagnement des gamètes de carpe. Diplôme, Fin d'études Institute Superieur Agricole de Beauvais.

Plouidy, M. G., et R. Bullard. 1982. The chemical composition of the companion fluids of the gametes in the common carp (Cyprinus carpio). Dans Reproductive physiology of fish. Proceedings of the International Symposium on Reproductive Physio- logy of Fish, 2-6 August 1982, Wageningen, The Netherlands. Éditeurs: C. J. J. Richter et H. J. Th. Goos. Centre for Agricultural Publishing and Documentation, Wageningen.

Roubaud, P., ct C. PAIRAUlt. 1980. Mcmbrane differentiation in the pregastrula of the teleost, Brachydanio rerio Hamilton-Buchanan (Teleostei: Cyprinidae). A scanning electron microscopy study. Reprod. Nutr. Dev. 20: 1515-1526.

SolN, S. G. 1977. Some features of the development of the carp (Cyprinus carpio) under hatchery conditions. J. Ichthyol. (Engl. Transl.), 17: 759-769

TrojnAR, J. R. 1977a. Egg and larval survival of white suckers (Catostomus commersoni) at low pH. J. Fish. Res. Board Can. 34: $262-266$

1977b. Egg hatchability and tolerance of brook trout (Salvelinus fontinalis) fry at low pH. J. Fish. Res. Board Can. 34: $574-579$.

VON RAuEN, H. M. 1964. Biochemisches Taschenbuch. SpringerVerlag, Berlin. pp. 37-110.

WINNICKI, A., et C. CYKowsKA. 1973. New data on the mechanism of water uptake in salmon eggs. Acta Ichthyol. Piscatoria, 3: 3-9. 